\title{
Limitations of the Hodgkin-Huxley Formalism: Effects of Single Channel Kinetics on Transmembrane Voltage Dynamics
}

\author{
Adam F. Strassberg \\ Computation and Neural Systems Program, California Institute of Technology, \\ Pasadena, CA 91125 USA \\ Louis J. DeFelice \\ Department of Anatomy and Cell Biology, Emory University School of Medicine, \\ Atlanta, GA 30322 USA
}

A standard membrane model, based on the continuous deterministic Hodgkin-Huxley equations, is compared to an alternative membrane model, based on discrete stochastic ion channel populations represented through Markov processes. Simulations explore the relationship between these two levels of description: the behavior predicted by the macroscopic membrane currents versus the behavior predicted by their microscopic ion channels. Discussion considers the extent to which these random events underlying neural signals mediate random events in neural computation.

\section{Introduction}

Action potentials within the neuron arise from the time-variant and voltage-dependent changes in the conductance of the neural membrane to specific ions. Hodgkin and Huxley based their famous model of active membrane on an assumption that the ion permeation processes existing within the membrane can be approximated as both continuous and deterministic (Hodgkin and Huxley 1952). However, the permeation processes existing within active membrane are known to be neither continuous nor deterministic. Active membrane is studded with discrete ion channels undergoing random fluctuations between open and closed stable states (Hille 1992). There have been few studies of the relationship between these two levels of description, the discrete stochastic behavior of the microscopic ion channels versus the continuous deterministic behavior of their macroscopic membrane currents (Clay and DeFelice 1983). This paper investigates these two regimes of activity through a comparison of the standard membrane model, based on the continuous Hodgkin-Huxley equations, to an alternative membrane model, based 
on discrete ion channel populations represented through Markov processes. When both models are used to simulate the active membrane of the squid Loligo giant axon, the convergence of the alternative model to the standard model can be examined. Under certain conditions, the behavior predicted by the alternative model will diverge from the behavior predicted by the standard model. Under these conditions, simulations suggest that random microscopic behavior, such as single channel fluctuations, becomes capable of generating random macroscopic behavior, such as entire action potentials.

\section{Methods}

The neural membrane of a space-clamped squid giant axon is modeled with an equivalent electric circuit. The space-clamp technique removes the spatial dependence of the membrane voltage and so the axon becomes effectively equivalent to an isopotential patch of membrane. A simple lumped circuit model thus can interpret the electrical characteristics of the membrane. The macroscopic membrane conductances are represented by the conductive elements $g_{\mathrm{Na}}, g_{\mathrm{K}}$, and $g_{\mathrm{L}}$ and the transmembrane voltage $V_{m}$ behaves according to the equation:

$$
\frac{d V_{m}}{d t}=-\frac{1}{C_{m}}\left[\left(V_{m}-E_{\mathrm{L}}\right) g_{\mathrm{L}}+\left(V_{m}-E_{\mathrm{K}}\right) g_{\mathrm{K}}+\left(V_{m}-E_{\mathrm{Na}}\right) g_{\mathrm{Na}}-I_{\text {inject }}\right]
$$

Behavior predicted by the standard Hodgkin-Huxley equations for the time-variant and voltage-dependent membrane conductances $g_{\mathrm{Na}}$ and $g_{\mathrm{K}}$ is compared to behavior predicted by alternative descriptions for these conductances based on their underlying ion channel populations.

Ion channel activity is modeled well by Markov processes. Each channel is assumed to randomly fluctuate between only a finite number of discrete stable states. Transition probabilities between these stable states are assumed to depend on the present stable state and the present membrane voltage and to be independent of the duration for which this present stable state has been occupied. Such Markov assumptions are used to interpret the data from patch-clamp experiments on single ion channels. These data often are too limited to allow for the isolation of a single Markov kinetic scheme from the several alternative schemes (Strassberg and DeFelice 1992; Kienker 1989; Clay and DeFelice 1983; Conti et al. 1975; Hille 1992). For this simulation of the ion channel populations underlying the membrane conductances $g_{\mathrm{Na}}$ and $g_{K}$, the simplest noncooperative and serial schemes have been chosen from the set of schemes capable of generating the desired macroscopic behavior.

Llano et al. (1988) have patch-clamped voltage-gated potassium channels in the active membrane of the squid giant axon. These channels show a single open state with a potassium ion conductance of $\sim 20 \mathrm{pS}$ 
(Llano et al. 1988). The following Markov kinetic scheme will reproduce the observed microscopic potassium channel behavior:

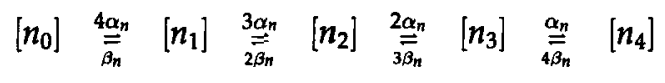

where $\left[n_{i}\right]$ refers to the number of channels within the population currently in stable state $n_{i}, n_{4}$ labels the single open state, and $\alpha_{n}$ and $\beta_{n}$ are the voltage-dependent rate constants from the Hodgkin-Huxley formalism (Armstrong 1969; Llano et al. 1988; Fitzhugh 1965; Hille 1992).

Vandenberg and Bezanilla (1988) have patch-clamped voltage-gated sodium channels in the active membrane of the squid giant axon. These channels show a single open state with a sodium ion conductance of $\sim 20$ pS (Bezanilla 1987; Vandenberg and Bezanilla 1988). The following Markov kinetic scheme will reproduce the observed microscopic sodium channel behavior:

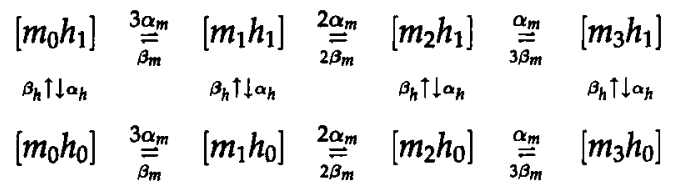

where $\left[m_{i} h_{j}\right]$ refers to the number of channels within the population currently in stable state $m_{i} h_{j}, m_{3} h_{1}$ labels the single open state, and $\alpha_{m}, \beta_{m}$, $\alpha_{h}$, and $\beta_{h}$ are the voltage-dependent rate constants from the HodgkinHuxley formalism (Bezanilla 1987; Vandenberg and Bezanilla 1988; Hille 1992; Fitzhugh 1965).

Simulation parameters are chosen to be identical to those values for squid axonal membrane used by Hodgkin and Huxley in their seminal paper (Hodgkin and Huxley 1952):

\begin{tabular}{llr}
\hline$C_{m}$ & Membrane capacitance & $1 \mu \mathrm{F} / \mathrm{cm}^{2}$ \\
$T$ & Temperature & $6.3^{\circ} \mathrm{C}$ \\
$E_{\mathrm{L}}$ & Leakage Nernst potential & $10.613 \mathrm{mV}$ \\
$g_{\mathrm{L}}$ & Leakage conductance & $0.3 \mathrm{mS} / \mathrm{cm}^{2}$ \\
$E_{K}$ & Potassium Nernst potential & $-12.0 \mathrm{mV}$ \\
$\bar{g}_{\mathrm{K}}$ & Maximal potassium conductance & $36 \mathrm{mS} / \mathrm{cm}^{2}$ \\
$D_{\mathrm{K}}$ & Potassium ion channel density & $18 \mathrm{channels} / \mu \mathrm{m}^{2}$ \\
$\gamma_{\mathrm{K}}$ & Potassium channel open state conductance & $20 \mathrm{pS}$ \\
$E_{\mathrm{Na}}$ & Sodium Nernst potential & $115.0 \mathrm{mV}$ \\
$\bar{g}_{\mathrm{Na}}$ & Maximal sodium conductance & $120 \mathrm{mS} / \mathrm{cm}^{2}$ \\
$D_{\mathrm{Na}}$ & Sodium ion channel density & 60 channels $/ \mu \mathrm{m}^{2}$ \\
$\gamma_{\mathrm{Na}}$ & Sodium channel open state conductance & $20 \mathrm{pS}$ \\
\hline
\end{tabular}

For a membrane model using discrete stochastic channel populations 
with the given Markov kinetics, 2.2 and 2.3, the potassium and sodium membrane conductances will satisfy

$$
g_{\mathrm{K}}(V, t)=\gamma_{\mathrm{K}}\left[n_{4}\right] \quad g_{\mathrm{Na}}(V, t)=\gamma_{\mathrm{Na}}\left[m_{3} h_{1}\right]
$$

For a membrane model using continuous deterministic Hodgkin-Huxley equations, the potassium and sodium membrane conductances will satisfy

$$
\begin{aligned}
g_{K}(V, t)=\bar{g}_{\mathrm{K}} n^{4} & g_{\mathrm{Na}}(V, t)=\bar{g}_{\mathrm{Na}} m^{3} h & \\
\frac{d n}{d t}=\alpha_{n}(1-n)-\beta_{n} n & \frac{d m}{d t}=\alpha_{m}(1-m)-\beta_{m} m & \frac{d h}{d t}=\alpha_{h}(1-h)-\beta_{h} h \\
\alpha_{n}=\frac{0.01\left(V_{m}+10\right)}{e^{(V m+10) / 110}-1} & \alpha_{m}=\frac{0.1\left(V_{m}+25\right)}{e^{\left(V_{m}+25\right) / 10}-1} & \alpha_{h}=0.07 e^{V_{m} / 20} \\
\beta_{n}=0.125 e^{V_{m} / 80} & \beta_{m}=4.0 e^{V_{m} / 18} & \beta_{h}=\frac{1}{e^{\left(V_{m}+30\right) / 10}+1}
\end{aligned}
$$

All simulations of membrane behavior are performed using GENESIS, ${ }^{1}$ an object-oriented general purpose neural simulator for the UNIX/Xwindows environment. Two new GENESIS objects are designed to model squid potassium and sodium ion channel populations undergoing Markov kinetics as given by 2.2 and 2.3 respectively. ${ }^{2}$ GENESIS scripts are produced for an isopotential patch of squid giant axon membrane under both the voltage-clamp and free-running experimental paradigm with membrane conductances represented through either Hodgkin-Huxley equations or channel populations with Markov kinetics.

\section{Results}

Figure 1 shows the voltage-clamp step response of the membrane conductances $g_{\mathrm{K}}$ and $g_{\mathrm{Na}}$. Both the continuous Hodgkin-Huxley equations and the discrete channel population Markov models are used alternatively to represent the membrane conductances. Note that as the size of each channel population is increased, the response from the discrete channel model converges to the behavior predicted by the continuous Hodgkin-Huxley currents. Figure 2 shows the response of a free-running membrane patch to a constant current injection and Figure 3 shows the resting response with no current injection. Figure 4 compares the mean firing frequencies of these responses to the membrane surface area of their underlying patches. For fixed channel densities, as the membrane surface area is increased, the response from the simulation of a constant density of ion channels converges to the response from the standard

\footnotetext{
${ }^{1}$ GENESIS (C1989 designed by Matt Wilson and Jim Bower at California Institute of Technology. Inquiries may be directed to genesis@caltech.edu or genesis@caltech.bitnet.

${ }^{2}$ Several new objects and scripts have been incorporated into the source code of GENESIS v1.4 as of July, 1992. Inquiries may be directed to strass@cns.caltech.edu.
} 

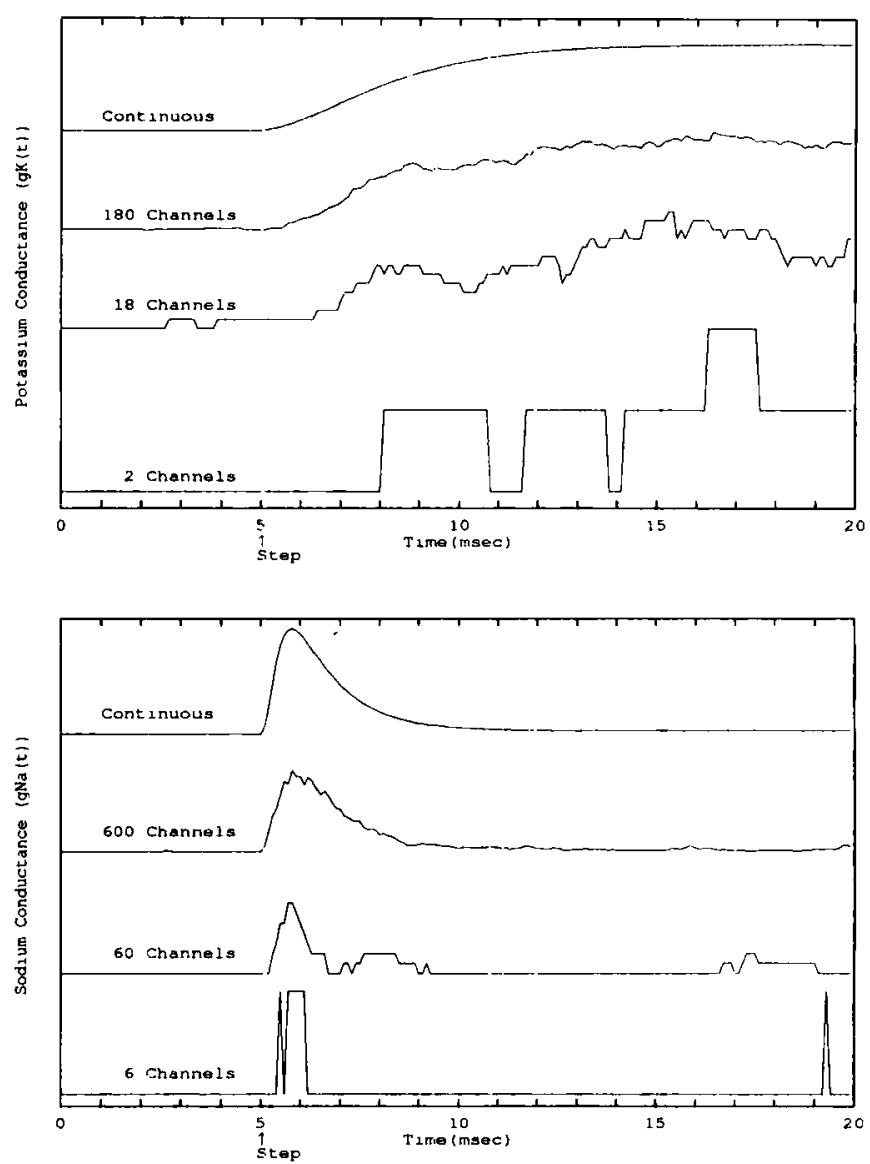

Figure 1: Voltage-clamp step response of membrane conductance. Membrane voltage is clamped to $V_{\text {rest }}$ and stepped to $V_{\text {rest }}+50.0 \mathrm{mV}$ at $t=5.0 \mathrm{msec}$. The response of each active membrane conductance is simulated with varying populations of discrete channels undergoing appropriate voltage-dependent Markov kinetics. These responses are compared to the behaviors predicted by the continuous Hodgkin-Huxley equations. Note that all outputs are normalized and displaced appropriately for display. As the size of each channel population is increased, the response from the discrete channel model converges to the behavior predicted by the continuous Hodgkin-Huxley equations, for both (top) the potassium conductance $g_{\mathrm{K}}(t)$ and (bottom) the sodium conductance $g_{\mathrm{Na}}(t)$. 


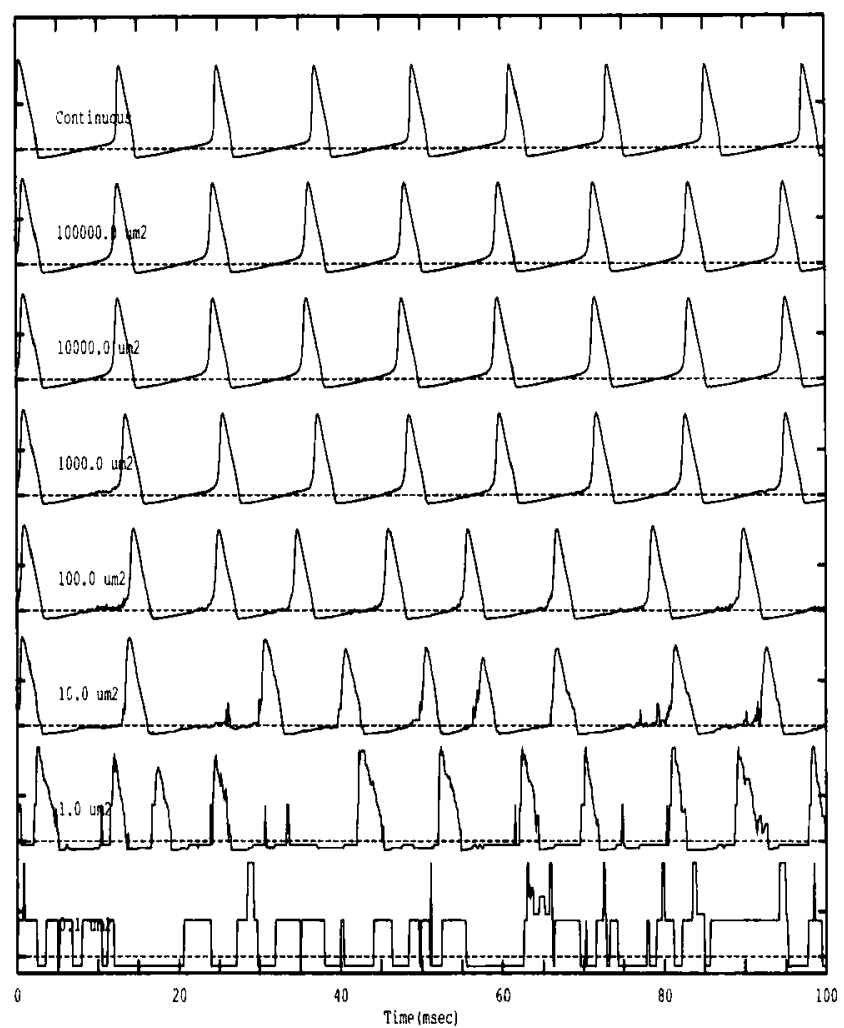

Figure 2: Membrane response with injection current. The membrane model is simulated with standard biophysical parameters for squid axonal membrane $\left(C_{m}, E_{\mathrm{Na}}, E_{\mathrm{K}}, E_{\mathrm{L}}, g_{\mathrm{L}}\right)$ and with constant current injection $\left(I_{\text {inject }}=100 \mathrm{pA} / \mu \mathrm{m}^{2}\right)$. The continuous Hodgkin-Huxley equations and the discrete channel populations are used alternatively to represent the membrane conductances $8 \mathrm{Na}$ and $8 \mathrm{~K}$. As the membrane surface area is increased, the response from the channel model converges to the response from the standard Hodgkin-Huxley model. Both models predict that a regular train of action potentials will occur when this constant current is injected. Note that, as the membrane surface area is decreased, the regularity of the spike train generated by the channel model diverges from the behavior predicted by the Hodgkin-Huxley model.

Hodgkin-Huxley model. Both models predict that, for large membrane surface areas, a train of action potentials will occur when constant current is injected and that no activity will occur when no current is injected. However, note that, as the membrane suface area is decreased, the be- 


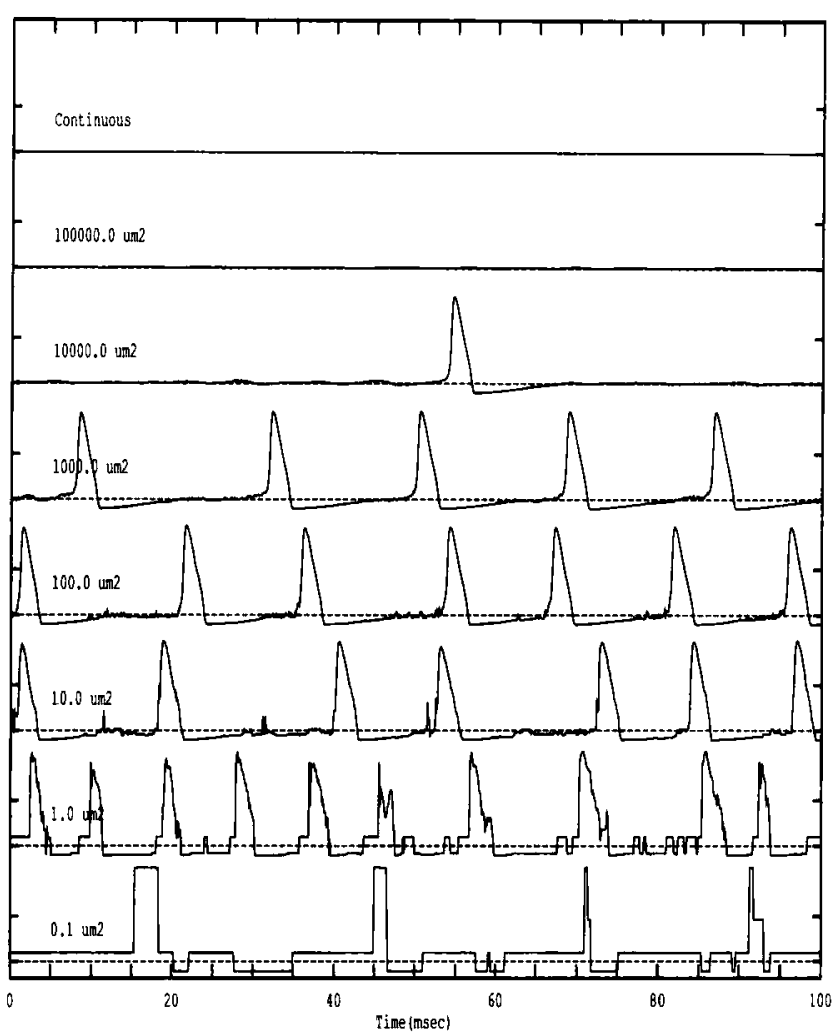

Figure 3: Membrane response without injection current. The membrane model is simulated with standard biophysical parameters for squid axonal membrane $\left(C_{m}, E_{\mathrm{Na}}, E_{\mathrm{K}}, E_{\mathrm{L}}, g_{\mathrm{L}}\right)$ and with no current injection $\left(I_{\text {inject }}=0 \mathrm{pA} / \mu \mathrm{m}^{2}\right)$. The continuous Hodgkin-Huxley equations and the discrete channel populations are used alternatively to represent the membrane conductances $g_{\mathrm{Na}}$ and $g_{\mathrm{K}}$. As the membrane surface area is increased, the response from the channel model converges to the response from the standard Hodgkin-Huxley model. Both models predict that no activity occurs when no current is injected. However, as the membrane surface area is decreased, the active behavior predicted by the channel model diverges dramatically from the lack of activity predicted by the Hodgkin-Huxley model.

havior predicted by the channel model diverges dramatically from the behavior predicted by the Hodgkin-Huxley model. These simulations suggest that, for an isopotential membrane patch with constant densities 


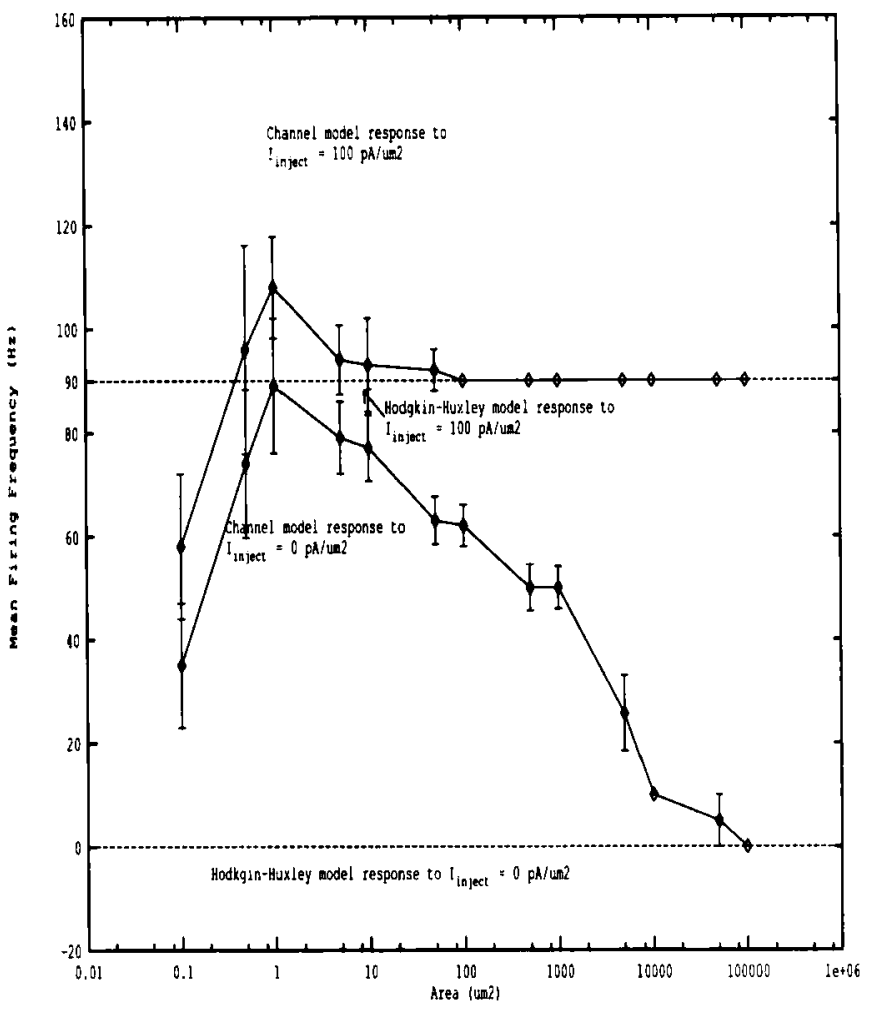

Figure 4: Mean firing frequency versus membrane area. For a given membrane area and a given constant current injection, the number of attendant action potentials is averaged over a $1 \mathrm{sec}$ duration to derive a mean firing frequency. As membrane area increases, the firing frequencies from the channel model converge to the firing frequencies from the Hodgkin-Huxley model. However, as membrane area decreases, these responses diverge dramatically. These simulations suggest that, as the area of an isopotential membrane patch is decreased, the voltage noise from single channel fluctuations will become capable of eliciting entire action potentials. (Over the smaller membrane surface areas, the graph shows the mean firing rates to first increase and then decrease. For such small regions, the opening of a single channel will depolarize the membrane to $E_{\mathrm{Na}}$ and so the definition of "action potential" becomes somewhat obfuscated.)

of sodium and potassium channels, as the membrane area is decreased, the fluctuations of single channels will become capable of eliciting entire action potentials. 


\section{Discussion}

The standard membrane model, based on the Hodgkin-Huxley equations, has been compared to an alternative membrane model, based on ion channel populations represented through Markov processes. When both models are used to simulate the active membrane of the squid Loligo giant axon, the explicit convergence of the alternative model to the standard model can be observed. However, under certain conditions, the behavior predicted by the alternative model diverges dramatically from the behavior predicted by the standard model.

\subsection{Membrane Voltage Perturbations Due to Single Ion Channel} Fluctuations. The divergent behavior can be explained through an analysis of the voltage perturbations across the membrane due to single ion channel fluctuations. Whenever a single ion channel moves from a closed state into an open state, the transmembrane voltage $V_{m}$ behaves according to the first-order transient:

$$
V_{m}(t)=\Delta V_{m}\left(1-e^{-\frac{t}{r}}\right)+V_{\text {rest }}
$$

The magnitude $\Delta V_{m}$ of the resultant voltage perturbation is mediated by a voltage divider between the conductance of the opened ion channel and the conductance of the surrounding membrane, which includes both the leakage conductance and the summed conductances of all other currently opened ion channels. The rise-time $\tau$ of this resultant voltage perturbation is equal to the membrane capacitance divided by the total membrane conductance. Note that there will be a correction term to the usual area-independent $\tau$ because the total membrane conductance is now the sum of both the conductance of the membrane surrounding the opened channel, which does scale with area, and the conductance of the individual opened channel, which does not scale with area.

For a given ion channel, the magnitude of the open state conductance and the voltage dependence of the stable state kinetics are independent of the surface area of the surrounding membrane. However, when this ion channel enters the open state, both the magnitude $\Delta V_{m}$ and the risetime $\tau$ of the resultant voltage perturbation across the membrane are dependent on the surface area of the surrounding membrane. For the specific biophysical parameters of squid axonal membrane, the voltage perturbation due to the random opening of a single sodium channel simplifies to

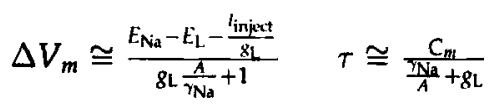

where $A$ is the total membrane surface area (Strassberg and DeFelice 1992). As this surface area $A$ is decreased, the magnitude $\Delta V_{m}$ increases 
of averaged data trials, which show much less variability. However, the fluctuations of single channels are probabilistic events with a high degree of variability from one trial to the next. With the current absence of any strong consensus on how the nervous system encodes information (beyond the level of sensory transduction), one is unable to distinguish strongly the "signal" from the "noise." The filtering and averaging of the data to remove the "noise" thus may be removing important components of the data. (Bower and Koch 1992).

Although the full prevalence and abundance of spontaneous action potentials are presently unknown, many potential roles for such spontaneous activations do exist in neural computation. While the effect of noise in a sensory system may be generally detrimental, the effect of noise in a planning, coordination, or motor system would not necessarily be as severe. For example, spontaneous action potentials could stop the repetition of unrewarded preprogrammed behaviors or perhaps even allow for the generation of entirely new responses to novel stimuli. During neurodevelopment, random activity could play a role in the coordination, correlation, and robust tuning of receptive field structures. From neuroethology, we know that organisms generate a host of spontaneous behavior patterns on the macroscopic level, thus it is reasonable to hypothesize that such spontaneous macroscopic behaviors arise from spontaneous microscopic behaviors.

This paper has used simulation and analysis to show that theoretical mechanisms exist for both the attenuation and the amplification of single channel noise. Experimental convention typically has ignored the underlying stochastic nature of the neuron in favor of the averaged neural response properties. However, as more physiological data on spontaneous activations do become available, the degree to which the random microscopic events underlying neural signals mediate random macroscopic events in neural computation will become more apparent.

\section{Acknowledgments}

This material is based on work supported under a National Science Foundation Graduate Fellowship and an NIH HL-27385. We would like to express our deep appreciation to Dr. Christof Koch for his comments and suggestions throughout the preparation of this manuscript. We also would like to thank Hsiaolan Hsu and Dr. Henry Lester for helpful insights.

\section{References}

Armstrong, C. M. 1969. Inactivation of the potassium conductance and related phenomena caused by quaternary ammonium ion injected in squid axons. J. Gen. Physiol. 54, 553-575. 
Bezanilla, F. 1987. Single sodium channels from the squid giant axon. Biophys.J. 52, 1087-1090.

Bower, J., and Koch, C. 1992. Experimentalists and modelers: can we all just get along? Tr. Neurosci. 15, 458-461.

Clay, J., and DeFelice, L. 1983. Relationship between membrane excitability and single channel open-close kinetics. Biophys. J. 42, 151-157.

Conti, F., DeFelice, L. J., and Wanke, E. 1975. Potassium and sodium ion current noise in the membrane of the squid giant axon. J. Physiol. (London) 248, 4582.

Fitzhugh, R. 1965. A kinetic model of the conductance changes in nerve membrane. J. Cell. Comp. Physiol. 66, Suppl., 111-117.

Franciolini, F. 1987. Spontaneous firing and myelination of very small axons. J. Theor. Biol. 128, 127-134.

Hille, B. 1992. Ionic Channels of Excitable Membrane, 2nd ed. Sinauer Associates, Sunderland, MA.

Hodgkin, A. L., and Huxley, A. F. 1952. A quantitative description of membrane current and its application to conduction and excitation in nerve. J. Physiol. (London) 117, 500-544.

Kienker, P. 1989. Equivalence of aggregated Markov models of ion-channel gating. Proc. R. Soc. London B 236, 269-309.

Koch, C., Zador, A., and Brown, T. H. 1992. Dendritic spines: Convergence of theory and experiment. Science 256, 973-974.

Llano, I., Webb, C. K., and Bezanilla, F. 1988. Potassium conductance of the squid giant axon. J. Gen. Physiol. 92, 179-196.

Segev, I., and Rall, W. 1988. Computational study of an excitable dendritic spine. J. Neurophys. 60, 499-523.

Strassberg, A. F., and DeFelice, L. J. 1992. Limitations of the Hodgkin-Huxley formalism. Computation and Neural Systems Program-Memo 14, California Institute of Technology.

Vandenberg, C. A., and Bezanilla, F. 1988. Single-channel, macroscopic and gating currents from $\mathrm{Na}$ channels in squid giant axon. Biophys. J. 53, 226a.

Received 16 March 1992; accepted 26 February 1993. 